\title{
Incidence and predictors of critical events during urgent air-medical transport
}

\author{
Jeffrey M. Singh MD MSc, Russell D. MacDonald MD MPH, Susan E. Bronskill PhD, \\ Michael J. Schull MD MSc
}

Previously published at www.cmaj.ca

$\infty \infty$

See related commentary by Isakov, page 569

\section{ABSTRACT}

Background: Little is known about the risks of urgent air-medical transport used in regionalized health care systems. We sought to determine the incidence of intransit critical events and identify factors associated with these events.

Methods: We conducted a population-based, retrospective cohort study using clinical and administrative data. We included all adults undergoing urgent air-medical transport in the Canadian province of Ontario between Jan. 1, 2004, and May 31, 2006. The primary outcome was in-transit critical events, which we defined as death, major resuscitative procedure, hemodynamic deterioration, or inadvertent extubation or respiratory arrest.

Results: We identified 19228 patients who underwent air-medical transport during the study period. In-transit critical events were observed in $5.1 \%$ of all transports, for a rate of 1 event per 12.6 hours of transit time. Events consisted primarily of new hypotension or airway management procedures. Independent predictors of critical events included female sex (adjusted odds ratio [OR] 1.3, $95 \%$ confidence interval $[\mathrm{Cl}] 1.1-1.5)$, assisted ventilation before transport (adjusted OR 3.0, 95\% CI 2.3-3.7), hemodynamic instability before transport (adjusted OR $3.2,95 \% \mathrm{Cl} 2.5-4.1)$, transport in a fixed-wing aircraft (adjusted OR 1.5, 95\% Cl 1.2-1.8), increased duration of transport (adjusted OR 1.02 per 10-minute increment, 95\% Cl 1.01-1.03), on-scene calls (adjusted OR 1.7, $95 \%$ CI 1.4-2.1) and type of crew (adjusted OR 0.6 for advanced care paramedics v. critical care paramedics, $95 \%$ Cl 0.5-0.7).

Interpretation: Critical events occurred in about 1 in every 20 air-medical transports and were associated with multiple risk factors at the patient, transport and system levels. These findings have implications for the refinement of training of paramedic transport crews and processes for triage and transport.
$\Lambda$ ir-medical transport is critical in regionalized health care systems to ensure access to distant specialized health care resources. Transport of acutely ill patients removes them from the hospital setting and may put them at risk for clinical deterioration. Although little is known about the risk of adverse clinical outcomes associated with transport, patients may deteriorate in transit because of progression of underlying diseases, the physical stress of transport, or care delivered before or during transport.

The reporting of adverse events and outcomes during transport is inconsistent, and reproducible predictors of in-transit adverse events have not been identified..$^{1-3}$ Skilled transport teams may avert clinical deterioration and adverse events through timely critical interventions; therefore, measurement of both clinical deterioration and resuscitative interventions (critical events) is important. ${ }^{4,5}$ Characterization of critical events could be used to reduce medical errors, improve processes related to equipment and transport, and optimize the triage and preparation of patients before transport and the matching of transport crews and resources to patients at highest risk.

Our goal was to determine the incidence of in-transit critical events during urgent air-medical transport and identify factors independently associated with these events.

\section{Methods}

\section{Study design}

We conducted a population-based retrospective cohort study using data from the provincial air-medical transport organization (Ornge) of Ontario. Ontario is Canada's second largest province, with a landmass of more than 1 million $\mathrm{km}^{2}$. Northern Ontario constitutes $87 \%$ of the land area of the province, although it contains less than $7 \%$ of the population. Ornge

From the Interdepartmental Division of Critical Care Medicine (Singh) and the Division of Emergency Medicine (MacDonald, Schull), Department of Medicine, University of Toronto; Ornge (Singh, MacDonald); and the Institute for Clinical Evaluative Sciences (Bronskill, Schull), Toronto, Ont.

Cite as CMAJ 2009. DOI:10.1503/cmaj.080886 
conducts more than 17000 air-medical transports annually using rotary-wing (helicopter) and fixed-wing aircraft with specially trained paramedic flight crews under the direction of a central transport medicine physician. Clinical data are recorded in-flight by transport crews, transcribed by data abstractors and validated by range and logic checks.

We included all air-medical transports of patients 18 years of age or older in the province of Ontario between Jan. 1, 2004, and May 31, 2006. We excluded nonurgent transports of patients to a convalescent facility or home, scheduled transports for medical appointments, treatments or repatriation and transports related to organ donation. We also excluded from the main analysis patients who were transported by primary care paramedic crews, because these crews are generally assigned low-risk patients and have a limited scope of resuscitative interventions (Appendix 1, available at www .cmaj.ca/cgi/content/full/cmaj.080886/DC1).

The research protocol for our study was approved by the Sunnybrook Health Sciences Centre Research Ethics Board.

\section{Data collection}

We obtained data on the clinical and demographic characteristics of patients. We ascertained the administration of assisted ventilation, including manual ventilation with a bagvalve-mask, from mechanical ventilation records. We defined baseline hemodynamic instability as a systolic blood pressure of less than $80 \mathrm{~mm} \mathrm{Hg}$ or a mean arterial pressure of less than $60 \mathrm{~mm} \mathrm{Hg}$ before departure from the sending site, or the administration of vasopressors before departure from the sending site. We assigned each transport to 1 of 7 diagnostic categories (respiratory, cardiovascular, trauma, neurologic, surgical, obstetrics and other medical diagnoses) based on coding of the primary diagnosis by the paramedic crew.

We also obtained transport-specific characteristics. We imputed the distance of straight-line transport from the postal codes of the sending and receiving institutions ${ }^{6,7}$ or from the transponder data of global-positioning-system units when available. We also used postal codes to determine the rural or urban status of the sending site using Statistics Canada Census indicators. ${ }^{8}$ To account for differences in population density, geography and medical resources, we classified origin of transport as either northern Ontario (rural and remote) or southern Ontario (more urban and dense) based on administrative boundaries (Local Health Integration Networks 13 and 14 of the Ontario Ministry of Health and Long-Term Care). We defined an on-scene call as a transport in which crews delivered acute care or resuscitation on arrival at a site outside a health care facility.

\section{Outcome measures}

The primary outcome was in-transit critical events, which we defined as death, major resuscitative procedures (Box 1), hemodynamic deterioration (systolic blood pressure $<80 \mathrm{~mm} \mathrm{Hg}$, mean arterial pressure $<60 \mathrm{~mm} \mathrm{Hg}$ or in-flight administration of a vasopressor), inadvertent extubation, or respiratory arrest. The event must have occurred between the time of departure from the sending facility and the time of arrival at the receiving facility. We selected the composite
Box 1: List of major resuscitative procedures included in the primary outcome of in-transit critical events

Airway procedure

- Needle thoracostomy

- Needle or surgical cricothyroidotomy

- Nasotracheal intubation

- Orotracheal intubation

- Removal of foreign object

- Other Advanced Life Support airway

- Laryngeal mask airway

- Ascherman chest seal

Cardiac procedure

- Cardiopulmonary resuscitation or chest compressions

- Cardioversion

- Carotid sinus massage

- Precordial thump

- Manual defibrillation

- Automatic defibrillation

- Transcutaneous pacing

- Administration of dextrose or glucagon

outcome to represent a fatal or life-threatening clinical deterioration. We also recorded the number of discrete critical events involving the same patient during a single transport.

\section{Statistical analysis}

We generated descriptive statistics with planned secondary analyses stratified according to sex, diagnostic category, type of sending site (e.g., acute care hospital) and geography. We assessed variables for collinearity. We used multivariable logistic regression to identify factors that were independently associated with in-transit critical events. We decided that age and sex had sufficient importance to include them in the multivariable model. We selected all other candidate variables for multivariable analysis using a threshold for inclusion of $p<0.25$ from a $\chi^{2}$ test or univariable logistic regression as appropriate. ${ }^{910} \mathrm{We}$ handled missing data by list-wise deletion, whereby we excluded the entire record from analysis if any single value was missing. We excluded variables that were missing more than $20 \%$ of observations. We included data from low-risk transports by primary care paramedics in a sensitivity analysis to assess their impact on our principal findings. We used the Kruskal-Wallis test for medians and $\chi^{2}$ test for binary and categorical variables for comparisons across groups.

\section{Results}

We identified 49986 transports of patients during the study period. We excluded 30758 (9173 transports of pediatric or neonatal patients, 10644 low-risk transports, 3256 land-based transports and 7685 transports by primary care paramedic crews), for a final cohort of 19228 urgent air-medical transports. Characteristics of patients and transports are shown in Table 1. Details of the cohort stratified by sex and diagnostic 
category are provided in Appendices 2 and 3 (available at www.cmaj.ca/cgi/content/full/cmaj.080886/DC1). Patient characteristics and case mix varied according to transport origin (data not shown); $64.4 \%$ of all transports originated in northern Ontario. Compared with those of southern origin, northern transports had significantly more surgical $(12.4 \% \mathrm{v}$. $5.4 \% ; p<0.001)$ and medical $(19.1 \%$ v. $11.6 \% ; p<0.001)$ diagnoses and fewer instances of baseline hemodynamic instability or assisted ventilation before transport $(5.4 \% \mathrm{v}$. $22.7 \% ; p<0.001)$. They were also significantly longer (median $266 \mathrm{~km}$ v. $98 \mathrm{~km} ; p<0.001$ ) and involved more frequent use of fixed-wing aircraft $(64.0 \%$ v. $8.0 \%$ in southern Ontario; $p<0.001)$. The majority of on-scene calls were by helicopter $(91.8 \%)$. Nearly all on-scene calls in southern Ontario were related to trauma, whereas those in northern Ontario reflected a broader range of diagnoses.

\section{Outcomes}

At least 1 critical event occurred during $981(5.1 \%)$ of the transports. Compared with patients who had no critical events, those who did were older (median age 58 [interquartile range 37-78] v. 53 [interquartile range 35-68]; $p<0.001$ ), had greater baseline hemodynamic instability $(16.9 \%$ v. $2.8 \%$; $p<0.001)$ and were more likely to have received assisted ventilation before transport $(27.1 \%$ v. $9.1 \% ; p<0.001)$. In-transit hemodynamic deterioration was the most frequent critical event $(3.2 \%, n=613)$, followed by major resuscitative procedure $(2.1 \%, n=413)$. New vasopressor medications were administered during 155 transports $(0.8 \%)$. Twelve deaths occurred during transport $(0.1 \%)$. The use of resuscitative procedures was commonly related to airway management (Table 2). The success rate for first-attempt intubation was $64.5 \%$ for oral intubations and $75.0 \%$ for nasal. Unintentional extubation was infrequent $(n=5)$. Among transports in which resuscitative procedures were administered, $290(70.2 \%)$ had only 1 resuscitative procedure and $27(6.5 \%)$ had 4 or more. We observed a total of 1435 discrete critical events, for a rate of 1 event per 12.6 hours of transport time.

The characteristics of critical events varied by diagnostic category and sex. The rate of critical events was significantly lower (2.5\%) among obstetric patients. Female and male patients had similar crude rates of in-transit critical events $(5.3 \%$ and $4.9 \% ; p=0.19)$. Women had a significantly higher rate of in-transit hemodynamic deterioration $(3.8 \%$ v. $2.7 \%$; $p<0.001)$ and lower rates of resuscitative procedures $(1.8 \%$ v. $2.5 \% ; p<0.001)$ compared with men. Rates of critical events were lower among transports of northern origin $(4.5 \%$ v. 5.7\% for those of southern origin; $p<0.001)$ and transports from nonacute care facilities $(3.9 \%$ v. $5.1 \%$ for acute care facilities and $6.7 \%$ for on-scene calls; $p<0.001$ ).

\section{Factors associated with critical events}

We selected 13 variables for multivariable analysis (Appendix 4, available at www.cmaj.ca/cgi/content/full/cmaj $.080886 / \mathrm{DC} 1)$. We excluded rural status and transport distance because more than $20 \%$ of the data for these variables was missing. The final model consisted of 13389 transports, of which 832 had in-transit critical events. Multiple
Table 1: Baseline characteristics of 19228 patients who underwent urgent air-medical transport in Ontario between Jan. 1, 2004, and May 31, 2006

\begin{tabular}{|c|c|}
\hline Characteristic & No. (\%) of patients* \\
\hline \multicolumn{2}{|l|}{ Patient } \\
\hline Age, median (IQR) & $53.0(35-68)$ \\
\hline Sex & $n=18866$ \\
\hline Female & $8609(45.6)$ \\
\hline Male & $10257(54.4)$ \\
\hline Diagnostic category & $n=18001$ \\
\hline Airway or respiratory disease & 1294 \\
\hline Cardiovascular & $5937(33.0)$ \\
\hline Neurologic & 2496 (13.9) \\
\hline Trauma & 1741 \\
\hline Obstetric & 1302 \\
\hline Surgical & $1806(10.0)$ \\
\hline \multirow[t]{2}{*}{ Other medical } & $3425(19.0)$ \\
\hline & $n=19228$ \\
\hline Hemodynamic instability at baseline & 673 (3.5) \\
\hline Assisted ventilation before transport & $1923(10.0)$ \\
\hline \multicolumn{2}{|l|}{ Transport } \\
\hline Distance, km, median (IQR) & $214(109-346)$ \\
\hline Duration, min, median (IQR) & $59.0(36-85)$ \\
\hline $\begin{array}{l}\text { Time on ground with patient, min, } \\
\text { median (IQR) }\end{array}$ & $17.0(10-32)$ \\
\hline Type of aircraft & $n=17819$ \\
\hline Fixed-wing & $7128(40.0)$ \\
\hline Helicopter & $10691(60.0)$ \\
\hline Type of paramedic crew & $n=19228$ \\
\hline Advanced care & $8460(44.0)$ \\
\hline Critical care & $10768(56.0)$ \\
\hline \multicolumn{2}{|l|}{ Contextual } \\
\hline Sending facility & $n=17868$ \\
\hline Acute care hospital & $12549(70.2)$ \\
\hline Non-acute care facility & $2638(14.8)$ \\
\hline On-scene call & $2681(15.0)$ \\
\hline Location of sending site & $n=15363$ \\
\hline Rural & $8573(55.8)$ \\
\hline Northern Ontariot & $9894(64.4)$ \\
\hline Time of transport & $n=19228$ \\
\hline Night & $7499(39.0)$ \\
\hline Weekend & $5768(30.0)$ \\
\hline Season & $n=19228$ \\
\hline Spring & $4383(22.8)$ \\
\hline Summer & $5268(27.4)$ \\
\hline Autumn & $5101(26.5)$ \\
\hline Winter & $4476(23.3)$ \\
\hline
\end{tabular}

Note: IQR = interquartile range.

*Unless stated otherwise.

tLocal Health Integration Networks 13 or 14 as defined by the Ontario Ministry of Health and Long-Term Care. 
patient- and transport-level factors were independently associated with in-transit critical events (Table 3). Female sex (adjusted odds ratio [OR] 1.3, 95\% confidence interval [CI] 1.1-1.5), administration of assisted ventilation before transport (adjusted OR 3.0, 95\% CI 2.3-3.7) and baseline hemodynamic instability (adjusted OR 3.2, 95\% CI 2.5-4.1) were all significantly and independently associated with in-transit critical events. Transport in a fixed-wing aircraft (adjusted OR 1.5, 95\% CI 1.2-1.8), duration of transport (adjusted OR 1.02 per additional 10 minutes, 95\% CI 1.01-1.03), onscene calls (adjusted OR 1.7, 95\% CI 1.4-2.1) and type of crew (adjusted OR 0.6 for advanced care paramedics v. critical care paramedics, 95\% CI 0.5-0.7) were also associated with in-transit critical events.

\section{Sensitivity analysis}

In a prespecified sensitivity analysis, we included transports by primary care paramedic crews, increasing the cohort to 26913 transports. Critical events occurred during $4.1 \%$ of transports, for a rate of 1 event per 24 hours of transport time. In multivariable analysis, the results were similar to those of the main model with the exception of an association between age of patient and the outcome (OR 1.005, 95\% CI 1.0011.010). Transports by primary care paramedics were much more frequent in northern Ontario $(37.9 \%$ v. $12.9 \%$ of transports in southern Ontario); also, they had low rates of in-transit critical events $(1.7 \%)$ and administration of resuscitative procedures $(0.3 \%)$.

Table 2: Major resuscitative procedures that occurred during the air-medical transports

\begin{tabular}{|lrl}
\hline Procedure & $\begin{array}{c}\text { No. (\%) of observed } \\
\text { procedures* } \\
n=751\end{array}$ \\
\hline Airway* & $197(26.2)$ \\
\hline Oral endotracheal intubationt & $101(13.4)$ \\
\hline Other advanced life support & $63(8.4)$ \\
\hline Needle thoracostomy & $28(3.7)$ \\
\hline Nasal endotracheal intubationt & $19(2.5)$ \\
\hline Ascherman chest seal & 5 & $(0.7)$ \\
\hline Laryngeal mask & 2 & $(0.3)$ \\
\hline Cricothyroidotomy & & \\
\hline Cardiac & $98(13.0)$ \\
\hline Cardiopulmonary resuscitation & $95(12.6)$ \\
\hline or chest compressions & $63(8.4)$ \\
\hline Administration of dextrose or glucagon & $50(6.7)$ \\
\hline Transcutaneous pacing & $13(1.7)$ \\
\hline Manual defibrillation & $12(1.6)$ \\
\hline Automatic defibrillation & $5(0.7)$ \\
\hline Cardioversion & & \\
\hline Carotid sinus massage & & \\
\hline
\end{tabular}

*Multiple resuscitative procedures may have been observed in a single transport.

tIncludes unsuccessful attempts at intubation.
We conducted 2 post hoc analyses. We stratified data by transport distance $(<125 \mathrm{~km}, 125-250 \mathrm{~km}$ and $>250 \mathrm{~km})$ and found that transports of less than $125 \mathrm{~km}$ had more patients who had baseline hemodynamic instability or required assisted ventilation before transport (particularly in trauma and helicopter transports) and more in-transit critical events (5.8\% v. $4.7 \%$ of transports of $125-250 \mathrm{~km}$ and $4.8 \%$ of transports of $>250 \mathrm{~km}$ ). The independent association between fixed-wing transports and critical events was reversed in the short-distance group (OR 0.48 for $125 \mathrm{~km}$ transports v. 1.56 for transports of $125-250 \mathrm{~km}$ and 1.16 for transports of $>250 \mathrm{~km}$ ). We also reran our analyses to include terms for distance and interaction between distance and aircraft type; the results were not significant.

\section{Interpretation}

We found that an in-transit critical event occurred in 1 in every 20 urgent air-medical transports of adult patients in a transport system covering a large geographic area. This rate is low but clinically important given the serious nature of these events and the challenging out-of-hospital environment in which they occurred. In-transit critical events were independently associated with female sex, use of mechanical ventilation or hemodynamic instability before transport, transport in a fixed-wing aircraft, duration of transport, on-scene calls and type of crew.

The incidence of critical events found in our study cannot be compared directly with rates reported in the literature, given the heterogeneity in the criteria for inclusion, reporting of data and definitions of outcomes in other studies. ${ }^{3,1-15}$ The rate we observed is similar to that reported in a study of outof-hospital transport by fixed-wing aircraft with comparably defined outcomes ${ }^{12}$ and higher than the rate in a smaller series from a single-centre organization. ${ }^{16}$ The rate of 1 critical event per 12.6 hours of transit time in our study is comparable to rates in other acute care fields of medicine ${ }^{4,17-19}$ Critical events comprised mainly hemodynamic deterioration and administration of major resuscitative procedures during transport. Procedures for airway management were frequently administered, and the rate of successful intubation we observed is consistent with rates reported in other studies. ${ }^{20-24}$

We identified several patient- and transport-level factors that were independently associated with in-transit critical events. Female sex was a significant factor even after adjustment for patient- and transport-level confounders. This association persisted in the sensitivity analysis that included transports with primary care paramedic crews. Evidence is growing that disparities exist between men and women in treatment, utilization of health care resources and outcomes across a wide range of diseases..$^{25-30}$ In our study, this observation may be attributable to differences between men and women in how diseases present, how they are treated and patterns of referral and transport-related requests. Systemic biases during assignment of transports could lead to delayed transport of women, with resulting physiologic instability and a higher risk of critical events. Alternatively, the criteria used to triage patients may underestimate the risk of deterioration among female patients. We observed that men were more often transported 
by critical care paramedic crews even though their unadjusted rate of in-transit critical events was not significantly different from that of women. Although conclusions about appropriate matching of patients to resources cannot be drawn, better understanding of the root causes of this observation may help optimize allocation of transport resources according to levels of acuity and transport risk of patients.

Although some critical events are likely unpredictable (e.g., unanticipated sudden cardiac arrest), many of the critical events we observed were related to airway management. Some of these events might have been prevented with better elective airway management before transport. In our study, elective intubation of a patient before transport was not a standardized or protocol-driven decision. Further study of this issue could inform policy development and crew training.

Table 3: Factors associated with in-transit critical events in multivariable logistic regression model

\begin{tabular}{|c|c|c|c|}
\hline \multirow[b]{2}{*}{ Factor } & \multicolumn{3}{|c|}{ Odds ratio ( $95 \%$ confidence interval) } \\
\hline & & Crude & Adjusted* \\
\hline \multicolumn{4}{|l|}{ Patient } \\
\hline Age (per 10-year increment) & 1.1 & $(1.0-1.1)$ & $1.0(1.0-1.1)$ \\
\hline Sex, female & 1.1 & $(1.0-1.2)$ & $1.3(1.1-1.5)$ \\
\hline \multicolumn{4}{|l|}{ Diagnostic category } \\
\hline Medical (reference) & 1.0 & & 1.0 \\
\hline Surgical & 1.1 & $(0.8-1.4)$ & $1.1 \quad(0.9-1.5)$ \\
\hline Respiratory & 1.6 & $(1.2-2.1)$ & $1.0(0.8-1.4)$ \\
\hline Cardiovascular & 1.4 & $(1.2-1.7)$ & $1.3(1.0-1.6)$ \\
\hline Neurologic & 1.1 & $(0.8-1.4)$ & $0.8 \quad(0.6-1.1)$ \\
\hline Obstetric & 0.6 & $(0.4-0.8)$ & $0.7 \quad(0.4-1.0)$ \\
\hline Trauma & 1.4 & $(1.1-1.7)$ & $1.2(0.9-1.6)$ \\
\hline Hemodynamic instability before transport & 3.7 & $(3.2-4.3)$ & $3.2(2.5-4.1)$ \\
\hline Assisted ventilation before transport & 6.5 & $(5.3-7.9)$ & $3.0(2.3-3.7)$ \\
\hline \multicolumn{4}{|l|}{ Transport } \\
\hline Duration (per 10-minute increment) & \multicolumn{2}{|c|}{$1.02(1.01-1.03)$} & $1.02(1.01-1.03)$ \\
\hline $\begin{array}{l}\text { Time on ground with patient, min, } \\
\text { median (IQR) }\end{array}$ & \multicolumn{2}{|c|}{$1.06(1.04-1.07)$} & $1.02(1.00-1.04)$ \\
\hline Fixed-wing aircraft (v. helicopter) & 0.8 & $(0.7-1.0)$ & $1.5(1.2-1.8)$ \\
\hline Advanced care crew (v. critical care crew) & 0.5 & $(0.5-0.6)$ & $0.6 \quad(0.5-0.7)$ \\
\hline \multicolumn{4}{|l|}{ Contextual } \\
\hline \multicolumn{4}{|l|}{ Sending facility } \\
\hline Acute care hospital (reference) & 1.0 & & 1.0 \\
\hline Non-acute care facility & 0.7 & $(0.6-0.9)$ & $1.1(0.9-1.4)$ \\
\hline On-scene call & 1.3 & $(1.1-1.6)$ & $1.7(1.4-2.1)$ \\
\hline \multicolumn{4}{|l|}{ Season } \\
\hline Winter (reference) & 1.0 & & 1.0 \\
\hline Spring & 1.1 & $(0.9-1.3)$ & $1.2(1.0-1.4)$ \\
\hline Summer & 1.0 & $(0.8-1.2)$ & $1.0(0.8-1.2)$ \\
\hline Autumn & 0.9 & $(0.7-1.1)$ & $0.9 \quad(0.7-1.1)$ \\
\hline
\end{tabular}

*Adjusted for age, sex, diagnostic category, presence of hemodynamic instability before transport, use of assisted ventilation before transport, duration of transport (in minutes), time on ground with patient before transport, type of aircraft, type of paramedic crew, type of sending facility and season.
Our data suggest that patients who have hemodyamic instability before transport should be given special consideration for pre-emptive airway management, given the strong association with in-transit critical events in this group.

Use of fixed-wing aircraft was associated with in-transit critical events. We hypothesize that this association was attributable to preferential assignment of these aircraft to patients in remote areas and to residual confounding in this patient population rather than to characteristics of the aircraft itself. Supporting this hypothesis was the reversal of this association in shortdistance transports in the sensitivity analysis, in which sicker patients with high-risk injuries were more often transported by helicopter. We also observed a $2 \%$ increase in the odds of critical events with every 10-minute increase in duration of transport, which would be expected with increasing flight times.

The association between higher levels of paramedic skill and higher rates of critical events was likely related to the assignment of patients who were at highest risk of deterioration to more advanced crews. The scope of practice of advanced care paramedics is similar to that of critical care paramedics, and we rarely observed the few procedures carried out uniquely by critical care paramedics (e.g., needle cricothyroidotomy constituted $0.3 \%$ of all observed procedures; $n=2$ ). The low rate of critical events among primary care transports may be related to the assignment of crew to low-risk patients or to ascertainment bias. The limited scope of practice of these basic paramedics censored them from many procedures included in our outcome measure.

Issues specific to jurisdiction and context are notable in our study. The geography of Ontario requires longer air-medical transports than those typically reported in the United States ${ }^{31}$ or Europe. ${ }^{32,33}$ In isolated areas, the levels of skill and training of paramedic transport crews may exceed that of the available local health providers. Thus, crews may deliver primary care and resuscitation "on scene" when picking up a patient. Requests for transport are almost never declined by Ornge because of acuity of illness; when indicated, even patients with the most unstable conditions are transported with aggressive resuscitation before and during transport.

\section{Strengths and limitations}

Our study has several strengths. It was population-based and involved the largest published cohort of transported patients. The procedures and clinical protocols used by Ornge Transport Medicine are 
similar to those of providers in other jurisdictions, enhancing the generalizability of our findings.

Our study was limited by the use of retrospective data. We were unable to obtain a patient-level score of severity of illness that would allow direct comparison of the incidence of critical events in our study to those reported for other acutely ill populations. Hemodynamic instability and need for assisted ventilation before transport provide only crude surrogate measures for severity of illness. We excluded almost one-quarter of the transports in the data set to limit the cohort to patients who were at highest risk of in-transit clinical deterioration. This large proportion of low-acuity transports is primarily related to a greater need for transport among patients in isolated communities to provide basic care and consultation. Indeed, none of the 10644 patients designated as low risk had baseline hemodynamic instability or were receiving assisted ventilation before transport; only $1.8 \%$ experienced an in-transit critical event, and no deaths occurred.

\section{Conclusion}

We found that a critical event occurred in transit in 1 in every 20 air-medical transports of adult patients. Most often, these events were the administration of resuscitative procedures or hemodynamic deterioration. Female sex, mechanical ventilation or hemodynamic instability before transport, transport in a fixed-wing aircraft, duration of transport, on-scene calls and type of crew were independently associated with in-transit critical events. These findings may be useful in the refinement of protocols for the optimal alignment of transport resources with patients at high risk of requiring in-transit resuscitation. Our results may also inform the training of paramedic transport crews and continuing education to address the most common scenarios they face. Better understanding of the patient-level predictors of adverse events may also allow for improvements in the preparation of patients by hospital staff before transport.

This article has been peer reviewed.

Competing interests: None declared.

Contributors: Jeffrey Singh conceived the study and is the guarantor for this paper. All of the authors contributed to the design of the study and the analysis and interpretation of the data. Jeffrey Singh drafted the manuscript, and Russell MacDonald, Susan Bronskill and Michael Schull provided critical revisions. All of the authors approved the final manuscript submitted for publication.

Funding: This study was supported by a grant from the Foundation for Air Medical Research and Education, and by funding from Ornge. The study sponsors were not involved in the design and conduct of the study, the collection, management, analysis or interpretation of the data, or the preparation, review or approval of the manuscript.

\section{REFERENCES}

1. Fan E, MacDonald RD, Adhikari NK, et al. Outcomes of interfacility critical care adult patient transport: a systematic review. Crit Care 2006;10:R6.

2. Bion JF, Edlin SA, Ramsay G, et al. Validation of a prognostic score in critically ill patients undergoing transport. Br Med J (Clin Res Ed) 1985;291:432-4.

3. Marx G, Vangerow B, Hecker H, et al. Predictors of respiratory function deterioration after transfer of critically ill patients. Intensive Care Med 1998;24:1157-62.

4. Choy CY. Critical incident monitoring in anaesthesia. Curr Opin Anaesthesiol 2008;21:183-6.
5. Kram R. Critical incident reporting system in emergency medicine. Curr Opin Anaesthesiol 2008;21:240-4.

6. Wilkins R. Automated geographic coding based on the Statistics Canada postal code conversion files, health analysis and measurement group. PCCF+ Version $4 \mathrm{H}$ user's guide. Ottawa (ON): Statistics Canada; 2007.

7. Ng E, Wilkins R, Perras A. How far is it to the nearest hospital? Calculating distances using the Statistics Canada Postal Code Conversion File. Health Rep 1993;5:179-88.

8. Statistics Canada Postal Code Conversion File (PCCF): reference guide - October 2005. Ottawa (ON): Statistics Canada; 2006. Cat no 92F0153GIE. Available: http://dsp-psd.tpsgc.gc.ca/Collection/Statcan/92F0153GIE/92F0153GIE2006001.pdf (accessed 2009 June 8)

9. Hosmer DW, Lemeshow S. Applied logistic regression. 2nd ed. New York (NY): Wiley; 2000.

10. Austin PC, Tu JV. Automated variable selection methods for logistic regression produced unstable models for predicting acute myocardial infarction mortality. J Clin Epidemiol 2004;57:1138-46.

11. Uusaro A, Parviainen I, Takala J, et al. Safe long-distance interhospital ground transfer of critically ill patients with acute severe unstable respiratory and circulatory failure. Intensive Care Med 2002;28:1122-5.

12. Dewhurst AT, Farrar D, Walker C, et al. Medical repatriation via fixed-wing air ambulance: a review of patient characteristics and adverse events. Anaesthesia 2001;56:882-7.

13. Duke GJ, Green JV. Outcome of critically ill patients undergoing interhospital transfer. Med J Aust 2001;174:122-5.

14. Ligtenberg JJ, Arnold LG, Stienstra Y, et al. Quality of interhospital transport of critically ill patients: a prospective audit. Crit Care 2005;9:R446-51.

15. Barillo DJ, Dickerson EE, Cioffi WG, et al. Pressure-controlled ventilation for the long-range aeromedical transport of patients with burns. J Burn Care Rehabil 1997;18:200-5.

16. Seymour CW, Kahn JM, Schwab CW, et al. Adverse events during rotary wing transport of mechanically ventilated patients: a retrospective cohort study. Crit Care 2008;12:R71.

17. MacDonald RD, Banks BA, Morrison M. Epidemiology of adverse events in air medical transport. Acad Emerg Med 2008;15:923-31.

18. Spittal MJ, Findlay GP, Spencer I. A prospective analysis of critical incidents attributable to anaesthesia. Int J Qual Health Care 1995;7:363-71.

19. Findlay GP, Spittal MJ, Radcliffe JJ. The recognition of critical incidents: quantification of monitor effectiveness. Anaesthesia 1998;53:595-8.

20. Tam R, Maloney J, Gaboury I, et al. Quality assurance of pre-hospital endotracheal intubation performed by advanced care paramedics in Ottawa, Canada. Ottawa $(\mathrm{ON})$ : Departments of Emergency Medicine and Pediatrics, University of Ottawa; Ottawa Base Hospital Program; Ottawa Paramedic Service; Chalmers Research Group, CHEO Research Institute; 2006. Available: www.ohri.ca/emerg/docs /OPALS-20061211/QA-ETI.pdf (accessed 2009 June 8).

21. Jacoby J, Heller M, Nicholas J, et al. Etomidate versus midazolam for out-of-hospital intubation: a prospective, randomized trial. Ann Emerg Med 2006;47:525-30.

22. Allen TL, Delbridge TR, Stevens MH, et al. Intubation success rates by air ambulance personnel during 12-versus 24-hour shifts: Does fatigue make a difference? Prehosp Emerg Care 2001;5:340-3.

23. O'Brien DJ, Danzl DF, Hooker EA, et al. Prehospital blind nasotracheal intubation by paramedics. Ann Emerg Med 1989;18:612-7.

24. Pearson S. Comparison of intubation attempts and completion times before and after the initiation of a rapid sequence intubation protocol in an air medical transport program. Air Med J 2003;22:28-33.

25. Schoen C, Simantov E, Gross R, et al. Disparities in women's health and health care experiences in the United States and Israel: findings from 1998 National Women's Health Surveys. Women Health 2003;37:49-70.

26. Eastwood JA, Doering LV. Gender differences in coronary artery disease. J Cardiovasc Nurs 2005;20:340-51.

27. Redberg RF. Gender differences in acute coronary syndrome: invasive versus conservative approach. Cardiol Rev 2006;14:299-302.

28. Bittner V. Perspectives on dyslipidemia and coronary heart disease in women J Am Coll Cardiol 2005;46:1628-35.

29. Lard LR, Huizinga TW, Hazes JM, et al. Delayed referral of female patients with rheumatoid arthritis. J Rheumatol 2001;28:2190-2.

30. Fowler RA, Sabur N, Li P, et al. Sex-and age-based differences in the delivery and outcomes of critical care. CMAJ 2007;177:1513-9.

31. Davis DP, Peay J, Serrano JA, et al. The impact of aeromedical response to patients with moderate to severe traumatic brain injury. Ann Emerg Med 2005; 46:115-22.

32. Biewener A, Aschenbrenner U, Rammelt S, et al. Impact of helicopter transport and hospital level on mortality of polytrauma patients. J Trauma 2004;56:94-8.

33. Di Bartolomeo S, Sanson G, Nardi G, et al. HEMS v. Ground-BLS care in traumatic cardiac arrest. Prehosp Emerg Care 2005;9:79-84.

Correspondence to: Dr. Jeffrey M. Singh, University of Toronto, University Health Network, 399 Bathurst St., McLaughlin Wing, 2nd floor, Rm. 411K, Toronto ON M5T 2S8; fax 416 603-1068; jeff.singh@uhn.on.ca 